Audiology

Neurotology
Audiol Neurotol 2006;11:269-275

DOI: $\underline{10.1159 / 000093525}$
Received: December 2, 2005

Accepted after revision: March 24, 2006

Published online: May 23, 2006

\title{
Phenotypic Characterization of DFNA24: Prelingual Progressive Sensorineural Hearing Impairment
}

\author{
Regie Lyn P. Santos ${ }^{a, f} \quad$ Franziska M. Häfner ${ }^{b}$ Patrick L.M. Huygen ${ }^{e}$ \\ Thomas E. Linder ${ }^{d} \quad$ Albert A. Schinzel ${ }^{c}$ Thomas Spillmann ${ }^{b}$ Suzanne M. Leal ${ }^{a}$ \\ ${ }^{a}$ Department of Molecular and Human Genetics, Baylor College of Medicine, Houston, Tex., USA; ${ }^{b}$ Department of \\ Otorhinolaryngology and Head and Neck Surgery, University Hospital of Zurich, ${ }^{C}$ Institute of Medical Genetics, \\ University of Zurich, Zurich, and ${ }^{\mathrm{d} D e p a r t m e n t}$ of Otorhinolaryngology and Head and Neck Surgery, \\ Cantonal Hospital of Lucerne, Lucerne, Switzerland; ${ }^{\mathrm{e} D e p a r t m e n t ~ o f ~ O t o r h i n o l a r y n g o l o g y, ~ U n i v e r s i t y ~ M e d i c a l ~ C e n t r e ~}$ \\ St. Radboud Nijmegen, Nijmegen, ${ }^{f}$ Genetic Epidemiology Unit, Department of Epidemiology and Biostatistics, \\ Erasmus MC Rotterdam, Rotterdam, The Netherlands
}

\section{Key Words}

Autosomal dominant nonsyndromic hearing impairment •

DFNA24 haplotype $\cdot$ Prelingual hearing impairment •

Progressive hearing impairment

\begin{abstract}
This article describes the hearing impairment (HI) phenotype which segregates in a large multi-generation Swiss-German family with autosomal dominant nonsyndromic HI. The locus segregating within this pedigree is located on chromosome 4q35-qter and is designated as DFNA24. For this pedigree, audiometric data on 25 hearing-impaired family members are available. It was demonstrated that within this kindred the $\mathrm{HI}$ is sensorineural, bilateral, prelingual in onset, and progressive throughout life. Age-related typical audiograms depict steeply down-sloping curves, with moderate high-frequency $\mathrm{HI}$ at birth, then steady progression to moderate $\mathrm{HI}$ in the low frequencies, severe $\mathrm{HI}$ at mid-frequencies and profound $\mathrm{HI}$ at high frequencies by age 70 . Annual threshold deterioration was $\sim 0.5 \mathrm{~dB} /$ year at $1-2 \mathrm{kHz}$ after correction for presbycusis.

Copyright $\odot 2006$ S. Karger AG, Basel
\end{abstract}

\section{Introduction}

Because of the extensive genetic heterogeneity in hearing impairment (HI), the importance of detailed phenotypic characterization for each gene or locus must be emphasized. Currently, for the approximately 120 loci which have been mapped for nonsyndromic HI, only 37 genes have been identified [Van Camp and Smith, 2005]. Finding the causative gene for each locus, documenting the functional mutations, and the estimation of populationspecific prevalences for each gene and/or mutation will ultimately help in genetic screening, clinical management and physiologic understanding of the inner ear. However, the process of gene finding is often hindered by huge genetic intervals from which to screen candidate genes and the lack of validation regarding the significance of sequence variants in the same gene from other families or populations.

The DFNA24 locus was first identified in a Swiss-German family with a history of autosomal dominant nonsyndromic HI which dates back to the mid-19th century [Häfner et al., 2000]. To date, the causative gene for this locus has not been identified, and no other family or pop-

Dr. Suzanne M. Leal

Department of Molecular and Human Genetics, Baylor College of Medicine

One Baylor Plaza, Alkek Building N1619.01

Houston, TX 77030 (USA)

Tel. +1 713798 4011, Fax +1 713798 4373, E-Mail sleal@bcm.tmc.edu 


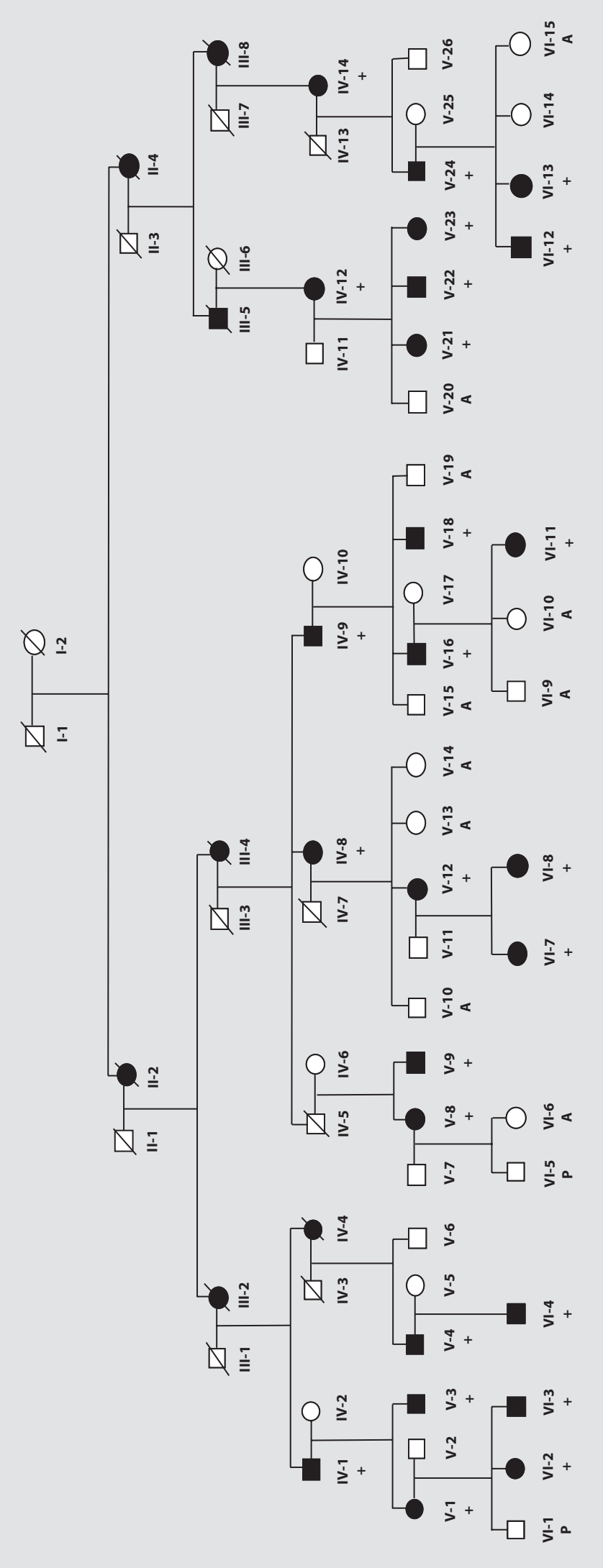

ulation has been linked to the same locus, inhibiting further fine-mapping efforts and validation of potential sequence variants. The aim of this study was to describe the audiometric phenotype associated with the DFNA24 locus, and hopefully aid in the identification of other families or individuals with the same phenotype that may also have HI due to DFNA24. Additionally, awareness of this phenotype could aid in the clinical management and genetic counseling of patients that have HI due to this locus.

\section{Materials and Methods}

Prior to data collection, the study was approved by the institutional review board of the University Hospital of Zurich. All procedures were carried out according to the Declaration of Helsinki. Informed consent was obtained from all adult study participants and parents of participants who were below 18 years of age.

The pedigree structure was reconstructed through interviews with family members (fig. 1). DNA and audiometric data were available for the last 3 of 6 generations. In total, 47 family members were genotyped ( 25 hearing-impaired and 15 hearing, plus 7 spouses), and of these individuals 38 had audiologic testing (25 hearingimpaired, 12 hearing, and 1 spouse V-25 who was homozygous for the $35 \mathrm{delG}$ variant of the GJB2 gene). Through careful medical history and physical examination, there was no evidence for the existence of syndromic abnormalities or vestibular disorders within the family, or that the HI was due to environmental risk factors.

For most family members, HI was first noted in early childhood while in primary school and fitted with hearing aids. At least 8 individuals in generations $\mathrm{V}$ and VI were diagnosed and fitted with hearing aids at ages 3-7 years (born 1957-1991), while 4 family members were known to be hearing-impaired in childhood but were fitted with hearing aids in adulthood (born 1922-1953, with ages at first hearing aid at 33-62). Individual V-18 was noted to be hearing-impaired while in kindergarten but started using amplification in high school. Eleven individuals received education in regular schools. Only 3 family members attended schools for the deaf. There is no evidence of anticipation within the pedigree because there was not a greater severity of $\mathrm{HI}$ or earlier age of onset among individuals in later generations. The earlier use of hearing aids in later generations is most likely due to advances in screening for $\mathrm{HI}$ and hearing aid technology.

Fig. 1. Pedigree drawing of DFNA24 family. The order of birth and gender of some individuals were modified to protect the family's anonymity. Filled symbols indicate HI, while clear symbols denote normal hearing or high-frequency impairment due to presbycusis. Family members who carry the DFNA24 haplotype are indicated by a + sign. Audiograms were available for all hearing-impaired individuals and for hearing family members that are marked as A. Two individuals marked with $\mathrm{P}$ have normal hearing based on pediatric audiology. 
Spouse V-25 is homozygous for the $35 \mathrm{delG}$ variant in the GJB2 gene, thus her affection status was made 'unknown' for the linkage analysis. Her children were also tested and they were heterozygous for $35 \mathrm{delG}$, therefore the $\mathrm{HI}$ in 2 out of 4 children who inherited the DFNA24 haplotype was assumed to be due to DFNA24 and not GJB2. The 6 other spouses who were genotyped but did not have audiograms were assumed 'unaffected' in the linkage analysis.

The genetic analysis for the family under study is reported elsewhere [Häfner et al., 2000]. A maximum multipoint LOD score of 11.6 was obtained at marker D4S1652. The linkage interval was between markers GATA129D03 and D4S1523/UT1366 at chromosomal region 4q35-qter, and the DFNA24 haplotype was found within the same linkage interval. This corresponds to a genetic length of $7.3 \mathrm{cM}$ according to the Rutgers combined linkage-physical map [Kong et al., 2004] and contains $8.1 \mathrm{Mb}$ based on Build 34 of the human reference sequence [International Human Genome Sequence Consortium, 2001]. All individuals who carry the DFNA24 haplotype are marked in the pedigree drawing with a + sign (fig. 1).

Results of previous audiometric testing were collected when possible. For 36 family members, audiometric measurements were performed in a soundproof compartment under ISO standards, at frequencies $0.125-8 \mathrm{kHz}$ for air conduction and $0.25-4 \mathrm{kHz}$ for bone conduction. Selected individuals underwent tympanometry, stapedius reflex and acoustic reflex testing. Three affected relatives were found to have concomitant middle ear problems. Stapedius reflex and acoustic reflex testing indicated loss of inner ear function in a few affected individuals who were tested.

For the audiogram analyses, immeasurable air conduction levels were fixed at an arbitrary value of $130 \mathrm{~dB}$. After a visual check on symmetry of the last-visit audiograms of all the affected individuals (fig. 2), the binaural mean air conduction threshold was calculated and used for further analyses. These analyses comprised linear regression analysis of threshold on age for each sound frequency. It was tested whether the slope of the regression line, referred to as annual threshold deterioration (ATD), expressed in $\mathrm{dB}$ per year, differed significantly from zero. To test if the intercept differed significantly from zero, it was checked whether the estimated $95 \%$ confidence interval (95\% CI) for the Y-intercept did not include zero. The test for significant slope was repeated after 'correcting' the air conduction threshold data by subtracting the median (P50) threshold for normal presbycusis according to the patient's age and sex as indicated by the ISO 7029 norm [ISO 7029, 1984]. The relative prevalence of significant slopes found among the total number of evaluated frequencies was used to test whether this prevalence was significantly high (i.e. $\mathrm{p}<0.05$ ) according to the appropriate binomial distribution. As outlined in a previous report [Huygen et al., 2003], the derived regression equations were used to obtain air conduction threshold estimates by age in decades in order to construct age-related typical audiograms (ARTAs).

\section{Results}

The audiogram data showed that the HI due to DFNA24 is sensorineural. Air-bone gaps $(>15 \mathrm{~dB}$ averaged at $0.5-$ $2 \mathrm{kHz}$ ) were noted only in 3 individuals whose last-visit audiograms were taken in childhood; in all 3 family mem- bers, a middle ear problem suggestive of effusion was documented. The audiograms from these 3 individuals were excluded from further analyses.

A random selection of individual last-visit audiograms is presented in figure 2. Both ears in all cases showed fair symmetry in air conduction threshold (fig. 2), so that the binaural mean threshold could be used as a key parameter. It is clear that HI, most pronounced at the higher frequencies, was already present at the youngest ages, and showed progressive deterioration at all frequencies with advancing age. Across all ages, the shape of the audiogram remained down-sloping, with greater affectation of the higher frequencies compared to the lower and middle frequencies.

Figure 3 shows the cross-sectional regression analysis of air conduction threshold on age. Threshold saturation played a role at frequencies of $>3 \mathrm{kHz}$. The plots in figure 3 show a gradual increase in threshold with advancing age. The ATD increased from $\sim 0.5-0.6 \mathrm{~dB} /$ year at $0.125-$ $0.5 \mathrm{kHz}$ to $\sim 0.9 \mathrm{~dB} /$ year at $4-8 \mathrm{kHz}$. The Y-intercept differed significantly from zero at all frequencies above $0.125 \mathrm{kHz}$; estimated values increased from $4 \mathrm{~dB}$ at $0.125 \mathrm{kHz}$ to $65 \mathrm{~dB}$ at $8 \mathrm{kHz}$ (fig. 3). In other words, all frequencies were affected in individuals with HI due to DFNA24, presumably at birth, but hearing at the higher frequencies was more severely impaired from the start and deteriorated at a more rapid rate. Unfortunately, there was an insufficient number of longitudinal data available to allow for reliable checks on individual progression.

To further determine whether there was progression of $\mathrm{HI}$ beyond presbycusis, cross-sectional linear regression analysis of the air conduction threshold minus the P50 for presbycusis for ages $<70$ years was performed for the frequencies $0.25,0.5,1,2,4$ and $8 \mathrm{kHz}$. The 'corrected' ATD varied from $\sim 0.2 \mathrm{~dB} /$ year at $0.25 \mathrm{kHz}$ to around $0.4-$ $0.5 \mathrm{~dB} /$ year at $1-2 \mathrm{kHz}$. At 4 and $8 \mathrm{kHz}$, the corrected ATD was only 0.4 and $0.2 \mathrm{~dB} /$ year, respectively (data not shown). However, it should be realized that at the latter frequencies the $\mathrm{HI}$ in many individuals was beyond the measurement limits of the audiometer so that no corrected threshold values could be calculated from the crude threshold measurements that were out of scale. Therefore, there was an increasing bias associated with the loss of data at these frequencies (fig. 3). Progression beyond presbycusis was significant in two out of six frequencies ( 1 and $2 \mathrm{kHz}$ ), which implies a significantly high prevalence (binomial tail probability, $\mathrm{p}=0.009$ ). Therefore, progression beyond presbycusis was distinctly present at the frequencies for which reliable, unbiased evaluation 
VI-12, M 7.45 y
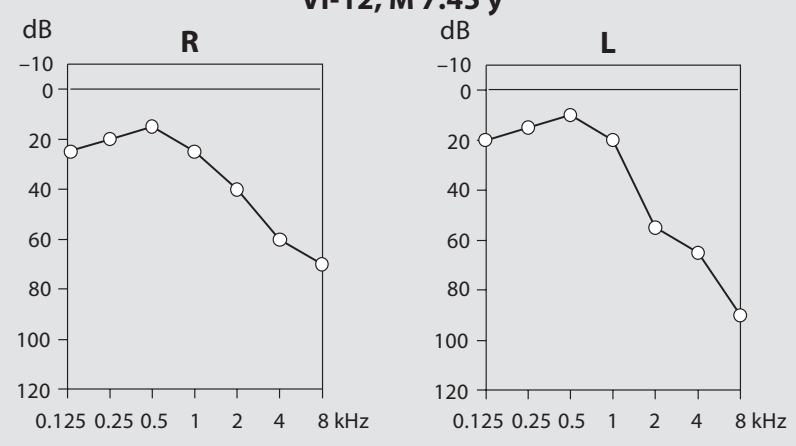

VI-3, M 17.15 y
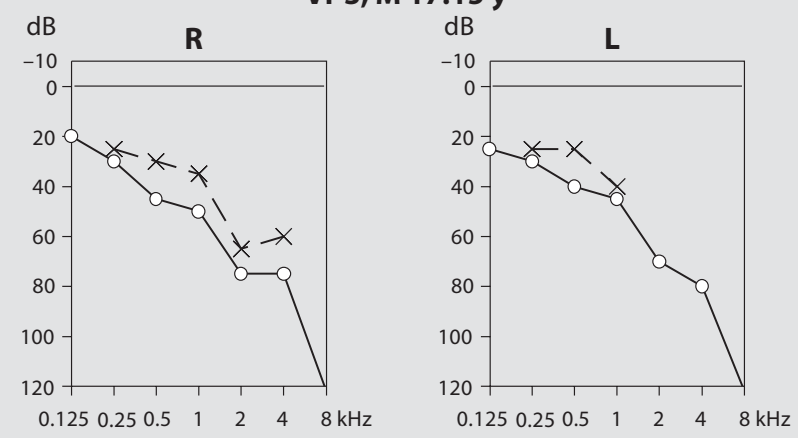

V-4, M 37.12 y
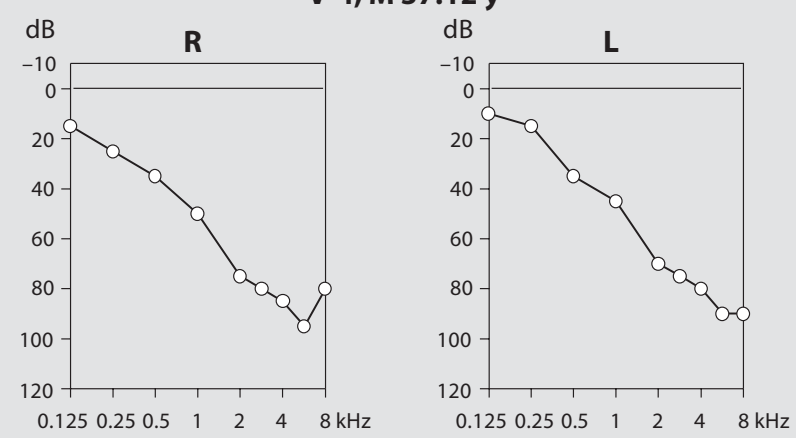

IV-14, F 61.09 y
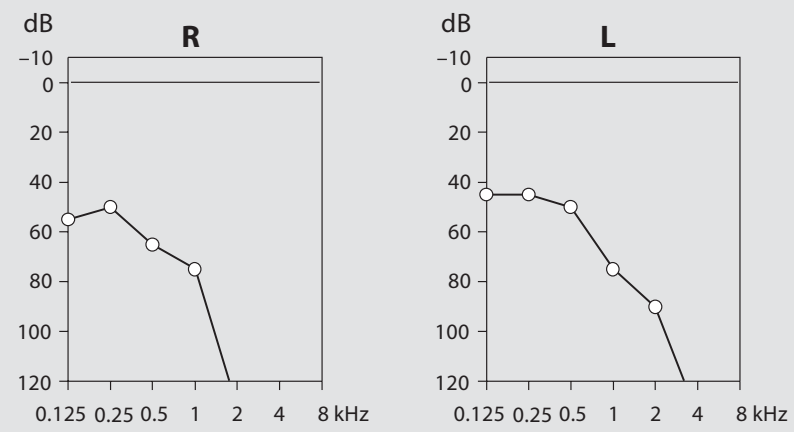

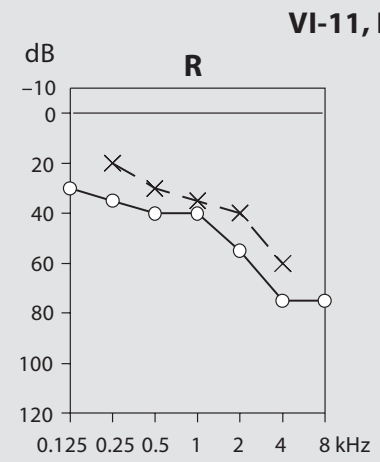

L

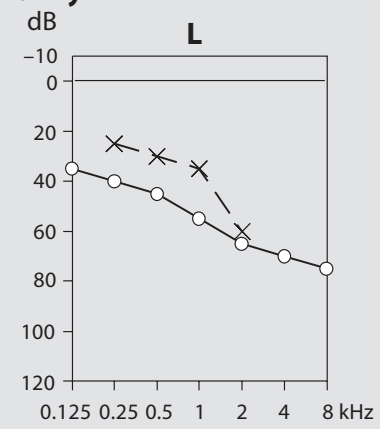

V-24, M 32.92 y
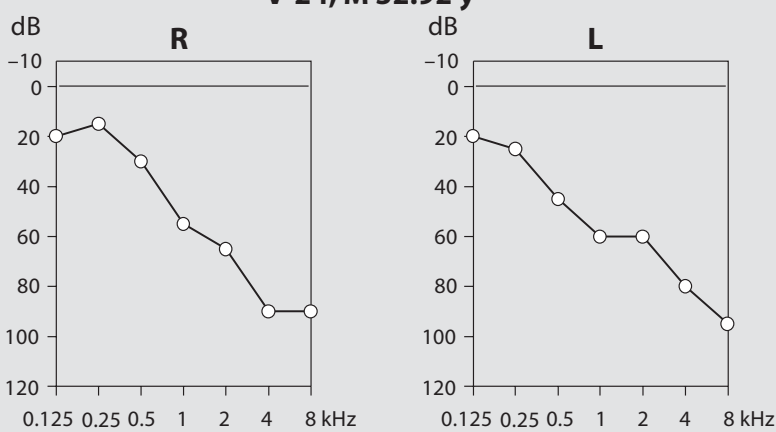

V-9, M 50.67 y
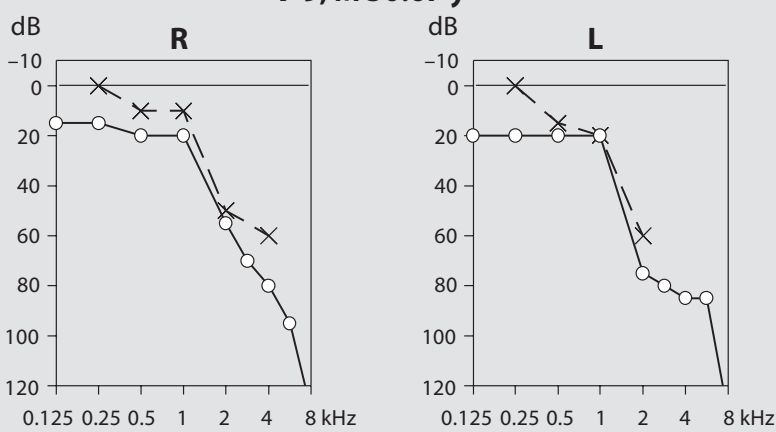

IV-9, M 76.01 y

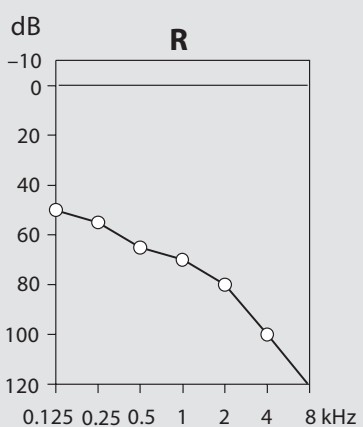

L

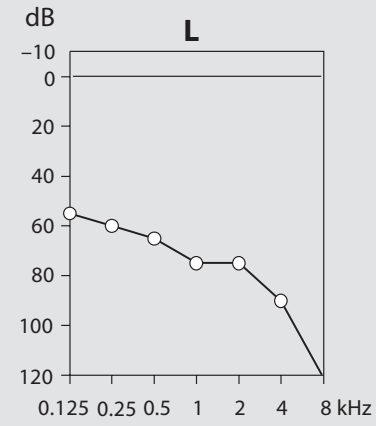

Fig. 2. Audiograms of a selection of hearing-impaired members of DFNA24 family ordered from top left to bottom right by age at last visit. These individuals were randomly selected by ordering all affected individuals by age and then choosing the audiogram from every 3rd person in the list. Above the audiogram panels, pedigree ID, gender $(\mathrm{M}=$ male; $\mathrm{F}=$ female $)$, age (in years) and laterality $(\mathrm{R}=$ right; $\mathrm{L}=$ left $)$ are indicated. Open circles denote air conduction thresholds (in $\mathrm{dB}$ ), crosses are bone conduction thresholds. 


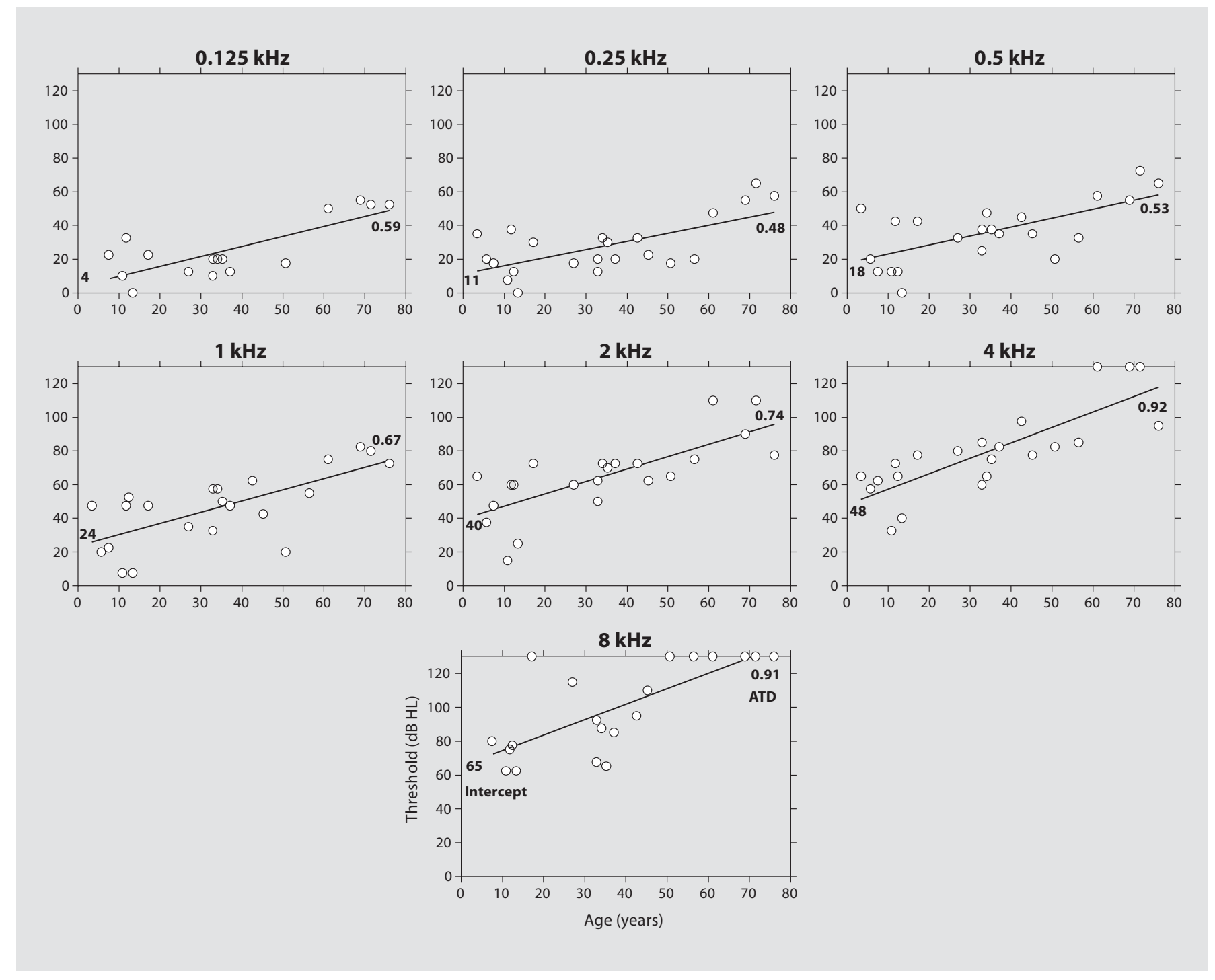

Fig. 3. Cross-sectional analysis of binaural mean air conduction threshold (dB HL) on age (in years) at the frequencies $0.125-8 \mathrm{kHz}$ for which more than 10 measurements were available. Each frequency panel includes the regression line with a bold figure on the right end indicating the ATD (dB/year) and a bold figure on the left end indicating the Y-intercept $(\mathrm{dB})$. All slopes and the Y-intercepts of $>4 \mathrm{~dB}$ are significantly different from zero.

could be achieved; however, deterioration in excess of normal presbycusis was limited to $\sim 0.5 \mathrm{~dB} /$ year at these frequencies.

Figure 4 shows the ARTAs that were derived from the regression results, and demonstrates that $\mathrm{HI}$ based on average hearing thresholds gradually progressed from mild $(\sim 10-30 \mathrm{~dB}$ at $0.25-1 \mathrm{kHz})$ to moderate $(\sim 40-60 \mathrm{~dB}$ at $2-8 \mathrm{kHz})$ impairment in childhood, to moderate $(\sim 40-$ $70 \mathrm{~dB}$ at $0.25-1 \mathrm{kHz})$ to profound $\mathrm{HI}(90 \mathrm{~dB}$ or more at $2-8 \mathrm{kHz}$ ) by age 70 . The degree of congenital $\mathrm{HI}$ that could be extrapolated from the cross-sectional regression analysis was significant and substantial, predominantly at the high frequencies (at age $0,>60 \mathrm{~dB}$ at $8 \mathrm{kHz}$ ).

Pure tone audiometry records were available for 10 out of 15 family members who were not spouses and were therefore at risk. Linkage analysis later revealed that these individuals did not carry the DFNA24 haplotype. For two other pedigree members who did not inherit the DFNA24 haplotype, normal hearing status is based on pediatric audiology (marked as $\mathrm{P}$ in fig. 1). For the remaining 3 non- 


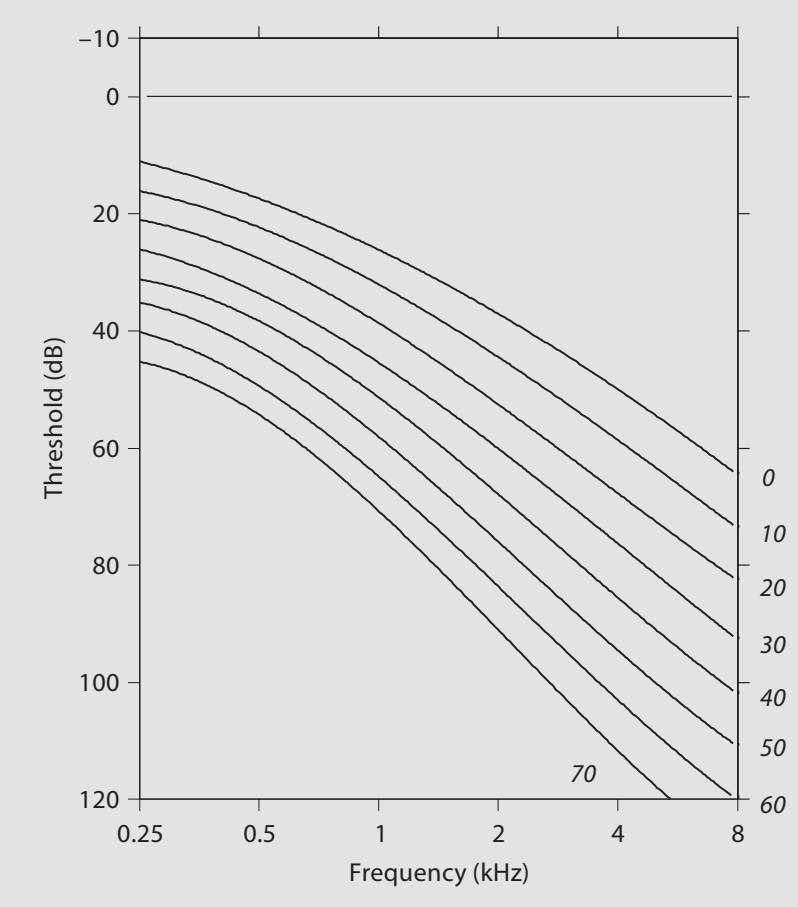

Fig. 4. ARTAs for the DFNA24 trait. Age (in years) is in italics on the right side of the audiogram curve.

carriers, audiometry has not been performed; based upon self-report, these individuals were assumed 'unaffected' in linkage analysis. Of those who had audiometric records, 4 noncarriers (aged 4-10) had normal hearing at all frequencies. In 6 individuals aged $>28$, some amount of $\mathrm{HI}$ (average threshold $20-48 \mathrm{~dB}$ or $0-28 \mathrm{~dB}$ hearing loss) was detected at the high frequencies $(4-12 \mathrm{kHz})$. However, testing based on P95 thresholds of ISO 7029 standards for presbycusis according to the individual's age and sex showed that these individuals did not have significant HI beyond presbycusis (data not shown). Therefore family members who did not carry the DFNA24 haplotype either had normal hearing $($ age $<10)$ or isolated high-frequency HI that may be attributable to presbycusis (ages 28-52). This is in contrast to carriers of the DFNA24 haplotype in which $\mathrm{HI}$ is detected at the mid-to-high frequencies by age 10 and at all frequencies by age 20 . As stated above, the $\mathrm{HI}$ in haplotype carriers is significantly greater than what is expected from presbycusis, particularly in the low frequencies.

\section{Discussion}

In summary, the DFNA24 locus is associated with HI that is sensorineural, bilateral and steeply down-sloping in audiometric configuration. When presbycusis was taken into account, there was a limited but significant degree of excess in hearing deterioration, amounting to $\sim 0.5 \mathrm{~dB}$ / year at $1-2 \mathrm{kHz}$, i.e. the frequencies that could be evaluated without tangible saturation bias. Thus it was established that, similar to most autosomal dominant nonsyndromic HI phenotypes, DFNA24 causes progressive deterioration of hearing with increasing age. However, the HI is prelingual, with moderate high-frequency loss expected at birth. The down-sloping shape of the audiogram is maintained throughout life, with steady progression to moderate impairment at the middle frequencies and severe impairment at high frequencies towards early adulthood, and then to moderate $\mathrm{HI}$ in the low frequencies, severe $\mathrm{HI}$ in mid-frequencies, and residual hearing at high frequencies in elderly age.

The DFNA24 phenotype clearly belongs to the category of high-frequency (down-sloping) progressive $\mathrm{HI}$, with the phenotypes of DFNA2, DFNA5 and DFNA7 being most similar. Eleven of 15 documented DFNA2 families harbor a mutation in the KCNQ4 gene (MIM 603537); the $\mathrm{HI}$ in most of these families was also presumably congenital and, after correction for presbycusis, had an ATD averaged for all frequencies at $0.65 \mathrm{~dB} /$ year [Topsakal et al., 2005]. DFNA5 and DFNA7 also have down-sloping audiometric shapes, but both have postlingual onset and much more rapid progression particularly in the first decades of life [De Leenheer et al., 2002; Huygen et al., 2002]. Therefore, of the three loci, the DFNA24 phenotype is closest in its description to that of DFNA2/KCNQ4, save for a seemingly slower progression rate of $\mathrm{HI}$ due to DFNA24. It is interesting to speculate whether the pathology involved in this group of high-frequency progressive $\mathrm{HI}$ phenotypes involves a common pathway, but there is no evidence available at present to forward such a hypothesis. Recently, it was suggested that dysfunction of the voltage-gated potassium channel KCNQ4 in the basal inner hair cells and spiral ganglion neurons affect electrical signaling in the inner ear [Beisel et al., 2005]. As regards to DFNA5, Dfna5-/- mice did not demonstrate significant HI or vestibular dysfunction [Van Laer et al., 2005]. The causative genes within the DFNA7 locus [Fagerheim et al., 1996] and the gene-poor DFNA24 region are yet unknown.

As more $\mathrm{HI}$ loci are mapped, the distinction among the different loci or genes in terms of functional effect is eas- 
ily blurred. It has been shown previously that reference to already published HI phenotypes, based on a specific audiometric pattern, can actually lead to faster genetic screening [Topsakal et al., 2005]. Detailed phenotypic description of more families or patients that are identified with a specific nonsyndromic HI locus will contribute to better clinical management, whether it be in the prevention of speech delay or disability through aggressive monitoring and rehabilitation after early diagnosis, in genetic counseling of affected families, or in greater awareness of environmental or occupational hazards that may compound an existing genetic susceptibility to development of HI. Because only the family reported here has been associated with DFNA24, there is a possibility that the phenotype has been influenced by genetic or environmental factors that are specific to this family and may therefore not exactly reflect the phenotype of other families or individuals that have HI due to the same gene or variant. It is hoped that the publication of the description of the DFNA24 phenotype will facilitate the reporting of other families with HI due to DFNA24 that will allow not only further fine-tuning of the phenotype but, more importantly, lead to the identification of a novel HI gene.

\section{Acknowledgements}

We thank the family members who participated in this study. This work was supported by the National Institutes of Health - National Institute of Deafness and Communication Disorders grant R01-DC03594 (to S.M.L.).

\section{References}

Beisel KW, Rocha-Sanchez SM, Morris KA, Nie L, Feng F, Kachar B, Yamoah EN, Fritzsch B: Differential expression of KCNQ4 in inner hair cells and sensory neurons is the basis of progressive high-frequency hearing loss. J Neurosci 2005;25:9285-9293.

De Leenheer EMR, van Zuijlen DA, Van Laer L, Van Camp G, Huygen PLM, Huizing EH, Cremers CWRJ: Clinical features of DFNA5. Adv Otorhinolaryngol 2002;61:53-59.

-Fagerheim T, Nilssen $\varnothing$, Raeymaekers P, Brox V, Moum T, Elverland $\mathrm{HH}$, Teig E, Omland $\mathrm{HH}$, Fostad GK, Tranebjærg L: Identification of a new locus for autosomal dominant non-syndromic hearing impairment (DFNA7) in a large Norwegian family. Hum Mol Genet 1996;5:1187-1191.

-Häfner FM, Salam AA, Linder T, Balmer D, Baumer A, Schinzel A, Spillmann T, Leal SM: A novel locus (DFNA24) for prelingual nonprogressive autosomal dominant nonsyndromic hearing loss maps to 4q35-qter in a large Swiss German kindred. Am J Hum Genet 2000;66: 1437-1442.
Huygen PLM, Bom SJH, Van Camp G, Cremers CWRJ: Clinical presentation of the DFNA loci where causative genes have not yet been cloned. DFNA4, DFNA6/14, DFNA7, DFNA16, DFNA20 and DFNA21. Adv Otorhinolaryngol 2002;61:98-106.

Huygen PLM, Pennings RJE, Cremers CWRJ: Characterizing and distinguishing progressive phenotypes in nonsyndromic autosomal dominant hearing impairment. Audiol Med 2003;1:37-46.

International Human Genome Sequence Consortium: Initial sequence and analysis of the human genome. Nature 2001;409:860-921.

ISO 7029: Acoustics: threshold of hearing by air conduction as a function of age and sex for otologically normal persons. Geneva, International Organization for Standardization, 1984.
Kong X, Murphy K, Raj T, He C, White PS, Matise TC: A combined linkage-physical map of the human genome. Am J Hum Genet 2004;75: 1143-1148.

Topsakal V, Pennings RJE, te Brinke H, Hamel B, Huygen PLM, Kremer H, Cremers CWRJ: Phenotype determination guides swift genotyping of a DFNA2/KCNQ4 family with a hot spot mutation (W276S). Otol Neurotol 2005; 26:52-58.

Van Camp G, Smith RJH: Hereditary hearing loss homepage. http://webhost.ua.ac.be/hhh/; accessed November 2005.

-Van Laer L, Pfister M, Thys S, Vrijens K, Mueller M, Umans L, Serneels L, Van Nassauw L, Kooy F, Smith RJ, Timmermans JP, Van Leuven F, Van Camp G: Mice lacking Dfna5 show a diverging number of cochlear fourth row outer hair cells. Neurobiol Dis 2005;19:386-399. 